\title{
L'apparentement : un mode de relation au monde humain et non humain que pourrait développer l'enseignement de la biologie
}

\section{Daniel Favre}

\section{(2) OpenEdition \\ Journals \\ Édition électronique \\ URL : http://journals.openedition.org/trema/2001 \\ DOI : 10.4000/trema.2001 \\ ISSN : 2107-0997 \\ Éditeur \\ Faculté d'Éducation de l'université de Montpellier}

Édition imprimée

Date de publication : 1 septembre 1996

Pagination : 45-52

ISSN : 1167-315X

\section{Référence électronique}

Daniel Favre, «L'apparentement : un mode de relation au monde humain et non humain que pourrait développer l'enseignement de la biologie », Tréma [En ligne], 9-10 | 1996, mis en ligne le 11 septembre 2013, consulté le 20 avril 2019. URL : http://journals.openedition.org/trema/2001 ; DOI : 10.4000/ trema.2001

Ce document a été généré automatiquement le 20 avril 2019.

Trema 


\title{
L'apparentement : un mode de relation au monde humain et non humain que pourrait développer l'enseignement de la biologie
}

\author{
Daniel Favre
}

\section{Introduction}

1 L'approche du courant psychologique américain représenté par H. SEARLES se pose comme une critique des modèles psychologiques européens. Ces derniers mettant trop l'accent, selon lui, sur le rôle structurant exercé par la relation avec les parents et pas assez sur la part attribuée dans la psychogenèse à la relation établie par un individu avec «l'environnement non humain ». En effet, l'environnement non humain, correspondant au monde non vivant et au monde vivant, est souvent traité comme étranger tant au développement de la personnalité qu'à celui des troubles psychiques. Or, l'homme n'est pas étranger au « monde non humain » mais un proche parent si on considère ses $98,4 \%$ d'ADN communs avec le chimpanzé; de plus, notre corps n'est-il pas lui-même «branché » en permanence avec le monde non humain?

2 Étudiant l'influence de cette relation sur le développement psychologique des humains, SEARLES (1960-1986) a pu caractériser ce qui correspondrait à la « maturité affective d'un individu par rapport à l'environnement non humain » qu'il a appelé : « apparentement » (relatedness).

3 La biologie est, en tant que discipline, celle qui aborde et décrit le plus directement les caractéristiques du monde non humain puisque ces angles d'approche s'étendent de la biochimie à l'étude des comportements. Son enseignement pourrait-il favoriser une mise en relation des élèves avec le monde non humain susceptible de participer au développement de leur maturité affective? 


\section{L'apparentement : un indicateur de maturité affective}

4 L'apparentement désigne, selon SEARLES, d'une part la perception d'une parenté intimecorrespondant psychique de la parenté structurelle (atomes, molécules, métabolismes, comportements...)- avec le monde non humain, et d'autre part le maintien de la conscience de son individualité en tant qu'être humain et de l'impossibilité de se fondre dans le monde non humain si étroitement qu'on soit lié à lui. Pour illustrer on pourrait considérer avec cet auteur que «si important que soit le niveau de relation qui nous fait voir dans un chat ou dans un arbre, par exemple, des porteurs de significations impliquant d'autres êtres humains- par déplacement et projection sur eux de nos sentiments inconscients, par transfert sur eux de nos attitudes relationnelles, par le jeu de diverses distorsions culturelles, etc.- il existe également un niveau où cette relation s'établit avec le chat en tant qu'il est un chat ou avec l'arbre en tant qu'il est un arbre " (op. cit., p. 39). La relation avec l'environnement peut donc s'établir à deux niveaux différents, complémentaires et nécessaires pour accéder à l'apparentement. La relation fusionnelle classiquement reconnue comme le premier mode de relation avec la mère s'étendrait ainsi également à tout l'environnement non humain, celui-ci jouerait donc également un rôle important dans la structuration de la personnalité. SEARLES suppose que si les récits relatant l'histoire de la vie sur la terre exercent sur nous une fascination et procurent un sentiment de bonheur, c'est peut-être parce qu'ils touchent une corde très profonde en lien avec nos expériences pré-verbales. Comme JUNG, il souligne la présence dans certains rêves de contenus qui n'ont de sens qu'en regard du développement phylogénétique.

5 Au cours de son développement psycho-physiologique l'enfant apprend à distinguer ce qui participe à son individualité (son corps, ses actes, ses pensées, ses émotions...) et le reste du monde. Cette distinction se réalise parallèlement au développement de la capacité d'abstraire, de se distancier de l'environnement non humain et de se le représenter sous forme de modèles conceptuels. Le développement individuel sera d'autant plus harmonieux et l'actualisation des potentialités personnelles importante que la relation avec l'environnement non humain a été complète. Cet environnement contribue selon SEARLES « de façon significative à la sécurité affective du nourrisson et de l'enfant, à la stabilité et à la continuité de son vécu et à l'élaboration de leur sentiment d'identité personnelle» (op. cit., p. 88). Contrairement à l'environnement humain, il fournit à l'enfant un terrain d'entraînement plus simple, plus stable et plus manipulable, pour développer des capacités de relations interpersonnelles et où il pourra prendre progressivement conscience de ses capacités et de ses limites (op. cit., p. 95). Au cours de l'adolescence, l'intérêt se déplace normalement du monde non humain vers l'environnement humain où les capacités précédemment développées vont pouvoir être réinvesties dans des relations d'amour interindividuelles. Au contraire, «les gens dont l'adolescence ne trouve pas son aboutissement continuent toute leur vie à se reconnaître davantage dans la nature que dans le genre humain. Avec la nature, ils se sentent dans une relation de parenté étroite et passionnée et avec leurs semblables dans une relation d'étrangeté, ce sont des misanthropes » (op. cit., p. 106).

6 Le concept d'apparentement va permettre de décrire les attitudes caractéristiques de la maturité affective vis à vis de l'environnement non humain. La maturité, dans ce type 
d'approche, ne cherche pas à se protéger ni contre le sentiment d'une parenté réelle avec la nature ni contre la conscience d'appartenir indéfectiblement à l'humanité, elle ne renonce pas aux frontières du moi (comme peuvent le proposer certaines approches mystiques) et ne se laisse pas leurrer par l'illusion de se fondre avec tel ou tel élément du milieu non humain (p. 108). Cette relation mature avec l'environnement non humain implique également une capacité à s'en distancier, pour entrer en relation avec quelque chose ou quelqu'un, il ne faut pas être lui ou fusionné avec lui. Quand cette distance optimale est installée peut alors naître un sentiment qui apaise l'appétit de toute puissance et favorise une acceptation plus totale de soi-même. L'apparentement constitue à travers le sentiment de stabilité et de continuité, que procure la relation au monde non humain, un antidote aux sentiments de l'absurde ou de l'insignifiance. La perception conjointe chez autrui des multiples liens qui le relie au monde non humain et de ce qui, dans sa spécificité humaine, le transcende, développe en nous la tolérance et l'acceptation à son égard.

7 Au contraire, l'expérience clinique montre à SEARLES qu'une relation insuffisamment développée avec le monde non humain se traduit à la fois par une dégradation du rapport que ces individus entretiennent ensuite avec leur environnement et par celle qu'ils tissent avec les autres humains. Ces humains ont alors tendance à compenser cette relation carencée avec le monde non humain à travers la possession de biens matériels auxquelles ils attribuent une importance excessive, et dont ils sont très dépendants, et à adopter une attitude faiblement responsable envers les processus de la vie (écologie, santé...).

8 L'enseignement des sciences naturelles et de la biologie en particulier représente souvent un des médiateurs principaux, surtout pour les populations très urbanisées, entre l'environnement non humain (les constituants de la lithosphère, de la biosphère et son propre corps) et l'individu. Il me parait dans ce cas pertinent de se demander si cet enseignement pourrait favoriser ou permettre en partie, l'accès à l'apparentement, à cette maturité affective qui, par ailleurs, comporte, comme on l'a vu de nombreux avantages, tels que l'approfondissement du sens de la réalité et une chance de mieux s'accepter et d'accepter les autres.

\section{L'enseignement de la biologie véhicule une image de la Vie et de l'Homme}

9 Les contenus d'enseignement d'une discipline sont rarement issus directement des travaux de recherche, ils font l'objet d'une mise en forme voire même d'une transformation suite à un "travail de didactisation» et à des choix axiologiques qui permettent aux "décideurs» de faire passer leurs valeurs (Develay, 1992). Les programmes, la manière de les interpréter par les enseignants peuvent ainsi aboutir à concevoir la vie et l'homme sous des angles spécifiques et réducteurs mais qui peuvent être en cohérence avec une manière de concevoir la société, les relations économiques, ainsi que la liberté individuelle et la finalité de l'existence. L'enseignement de la biologie pourrait-il participer à cette transmission de valeurs de manière clandestine, c'est à dire à l'insu des enseignants ?

10 L'étude des conceptions du systèmes nerveux chez les élèves à la sortie des classes terminales (BEC et FAVRE, $c f$ ces Actes), nous amène à penser que cet enseignement a 
contribué de manière sensible à accentuer le rôle du système nerveux dans le fonctionnement des réflexes et des mouvements visant l'action volontaire ou non, à sous estimer la participation du système nerveux dans la digestion, les processus cognitifs ou les rêves et surtout à ignorer son intervention dans la genèse des émotions ou des affects. Ces résultats confirment d'ailleurs l'analyse de RUMELHARD (1979) selon laquelle si l'accent est autant mis dans l'enseignement sur le réflexe ce serait parce que notre civilisation tend à privilégier la rapidité et ce mode de réactivité. Vraisemblablement, les enseignants ne participent pas volontairement à cette sélection des contenus enseignés et ont dû hériter leur vision de l'homme ou de la vie de ceux qui les ont formés. À titre d'exemple, le recueil des associations spontanées de mots autour du terme "cerveau » auprès d'un millier d'enseignants du secondaire et du primaire, nous a montré que si l'association se faisait avec "neurones" ou d'une manière générale avec le support biologique ou encore avec une métaphore mécanique, électrique ou informatique ou enfin avec la pensée, la réflexion et la logique, aucun n'associe spontanément le «cerveau » avec "émotion"; «peur»; " plaisir»; " panique» ou «jouissance». Pourtant, le premier indice intrasubjectif de la compréhension, chère aux enseignants, n'est-il pas une émotion fort plaisante?

11 On pourrait multiplier les interrogations de ce type à propos de la place importante réservée à l'enseignement de la théorie de la sélection naturelle, du rôle du hasard, du déterminisme génétique, de l'importance donnéeà la biologie moléculaire et inversement du peu d'importance accordé aux processus coopératifs et symbiotiques, aux interactions entre les organismes vivants, à l'interdépendance, à l'auto-organisation et à l'autonomie du monde vivant.

Une interprétation personnelle m'incite à penser qu'il est plus facile dans le premier cas d'imposer les valeurs majoritaires dites matérialistes du monde socio-économique actuel que dans le second. En tant qu'enseignant de biologie, je n'ai pas envie de propager des valeurs qui déresponsabilisent en donnant priorité au profit financier à court terme et en éludant les questions $\mathrm{du}$ sens et de l'incertitude que posent la perception et l'interprétation des phénomènes de la vie et de l'évolution. De plus, je trouve désagréable de me sentir utilisé à mon insu pour véhiculer des valeurs qui me paraissent incompatibles avec une des missions de l'enseignement : permettre le développement du libre-arbitre et de l'autonomie des apprenants. Cette prise de position très personnelle est rapportée ici surtout pour illustrer l'idée centrale de ce chapitre : l'enseignement de la biologie n'est pas neutre, il peut participer de manière plus ou moins consciente à l'élaboration de notre représentation du monde, de nous même et de notre interaction avec les autres.

Dans ce cas, ne serait-il pas souhaitable que l'enseignement de la biologie puissent contribuer à rencontrer le monde non humain en présentant explicitement les méthodes et les outils conceptuels (et autres modèles approximatifs et provisoires) pour se saisir abstraitement de ce monde d'une part et d'autre part des occasions de rencontrer le plus directement possible (par le biais des expériences sensorielles ou des jeux) les éléments de ce monde?

\section{Deux tendances pédagogiques à réconcilier}

L'analyse du discours pédagogique et scientifique d'un échantillon restreint d'enseignants de biologie du secondaire (Favre \& Rancoule, 1993),nous a permis de 
constater qu'un cours de biologie comprenait en moyenne quatre cents phrases énoncées en cinquante-cinq minutes contre vingt-cinq à trente phrases pour l'ensemble des élèves. $94 \%$ de ces phrases étaient au présent de l'indicatif et $86 \%$ avec le verbe être ». On a pu également remarquer l'absence complète des formules destinées à suspendre le jugement : « il me semble », « peut-être »... et du conditionnel. Dans ces conditions, je ne vois pas très bien comment le caractère ni approximatif ni provisoire des connaissances communiquées- et donc la non-possibilité de prendre du recul- permettrait aux élèves d'utiliser l'enseignement de la biologie pour développer leur sentiment d'apparentement.

De plus, lors des rares séances de travaux pratiques, la représentation qu'ont un grand nombre d'enseignants de la démarche expérimentale et scientifique semble également constituer une difficulté pour permettre aux élèves de nourrir leur relation à l'environnement non humain. Le filtre conceptuel que constitue le modèle de scientificité hérité sans doute, comme le signale STEWART (1995) (cf ces Actes), de la physique mécaniste, interpose entre le monde vivant et l'élève une sorte de barrière. Le monde humain et non-humain présenté par l'enseignement de la biologie apparaît ainsi trop souvent comme un monde étranger, rigide, excessivement à distance et abstrait, donc peu attrayant, et dont il faut mémoriser à court terme les ennuyeuses informations pour les restituer le jour du contrôle.

De multiples exemples, souvent ponctuels, suggèrent cependant que des pratiques différentes puissent exister, encore faudrait-il pouvoir les repérer et identifier ce qui dans ces pratiques pourrait alimenter les deux niveaux de relation que requiert l'apparentement. Dans le cadre de ces rencontres LAURET (1995) (cf. sa communication à ce Colloque) montre, par exemple, tout l'intérêt (motivation, mémorisation et compréhension accrues) qu'il y a à proposer aux étudiants un enseignement d'algologie en contact étroit avec le contexte environnemental dans lequel se développent les algues étudiées.

17 La modélisation suivante a été élaborée dans la perspective de fournir un moyen d'identifier des tendances pédagogiques présentes dans telle ou telle pratique. Nous faisons l'hypothèse que si ces tendances étaient utilisées conjointement elles pourraient faciliter l'accès à l'apparentement chez les apprenants de la biologie. 


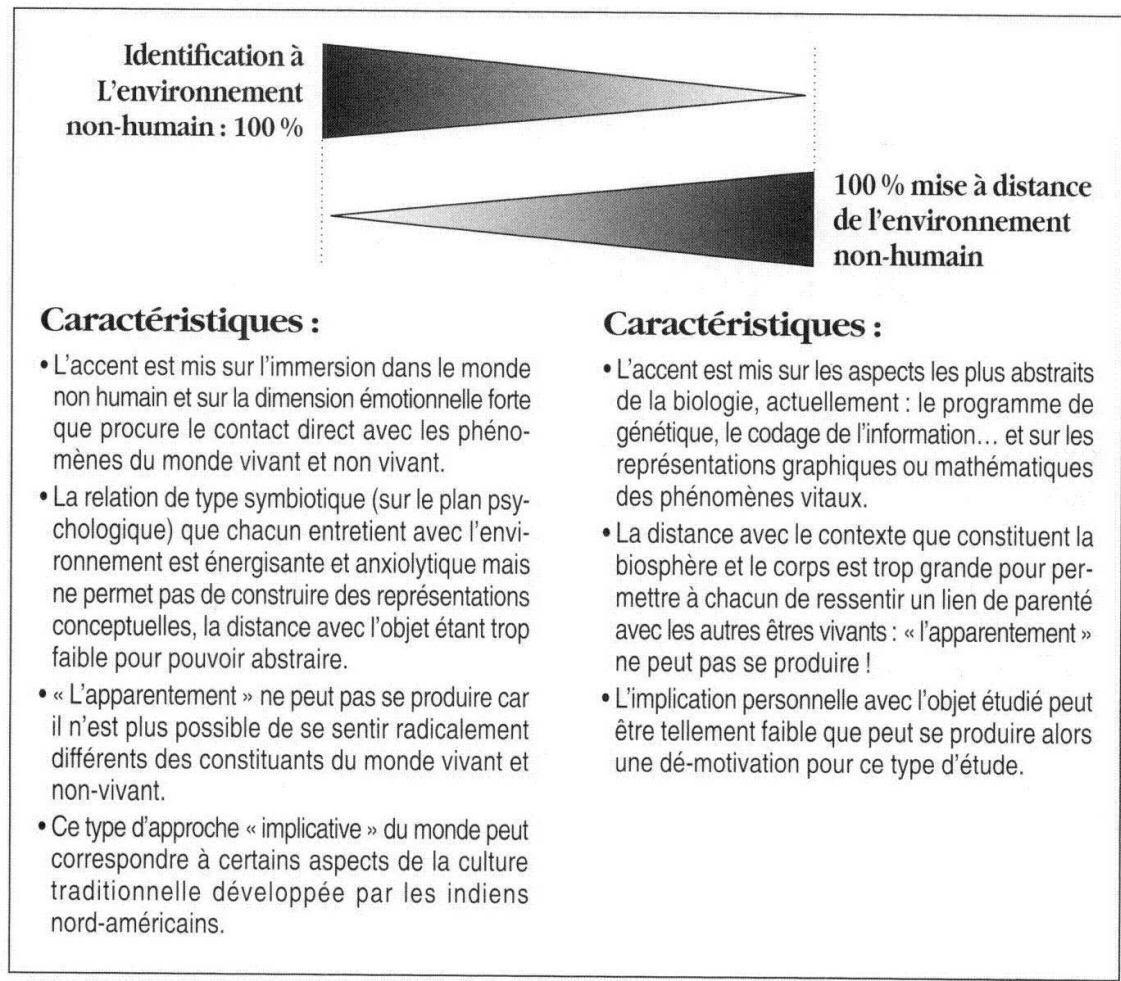

18 Pour chacun de ces deux pôles pris isolément, l'apparentement semble impossible: n'aurait-on pas intérêt, si l'on souhaite voir se développer chez les élèves et les étudiants une maturité affective descriptible en terme d'apparentement ainsi qu'une motivation accrue pour cette discipline, à inventer une forme d'enseignement qui associe plus étroitement ces deux tendances pédagogiques que les cultures ont eu tendance à opposer?

\section{Conclusion}

D'une manière générale, la question posée aux IV ${ }^{\text {es }}$ rencontres de didactique de la biologie à Cordoue (FAVRE, 1992) a conservé son actualité : à quoi pourrait bien servir la biologie pour les élèves qui ne se destinent ni à son enseignement, ni à la recherche dans les sciences de la vie?

20 Si la réponse consiste à énoncer la liste des connaissances de biologie réinvestissables dans le domaine de la santé ou de l'environnement, on s'aperçoit que l'enseignement de biologie comporte de nombreuses informations utiles pour mener à bien une existence de citoyen respectueux de son corps et de la biosphère. Cet enseignement comporte également des données de biologie moléculaire dont le réinvestissement dans la vie quotidienne me parait plus difficile. Le problème posé dans cet article, n'est pas de fournir des critères pour choisir les contenus utiles ou non de biologie à enseigner mais de s'interroger si la forme donnée à cet enseignement est susceptible de le rendre réinvestissable. Il me semble en lisant les travaux de SEARLES que les connaissances de biologie seraient plus facilement investies et réinvesties si l'enseignement permettait un plus grand développement de l'apparentement chez les apprenants.

21 L'accès à l'apparentement par la médiation de l'enseignement de la biologie paraît, en partie, possible si cet enseignement arrivait à la fois à nourrir notre lien de parenté avec 
le monde non humain mais aussi à nous en distancier suffisamment et non dogmatiquement pour devenir apte à nous percevoir comme radicalement différent de ce monde. L'excès de distanciation pourrait transformer la biologie en discipline ennuyeuse car trop coupée de ce qui fait sens pour soi, l'excès d'immersion dans l'environnement non humain ne conviendrait pas également à l'appropriation des connaissances, puisque de nombreux travaux de didactique ont clairement montré la faillite des enseignements de biologie fondés sur l'inductivisme.

Une des spécificités de l'enseignement de la biologie serait, outre de communiquer de manière moins ennuyeuse des connaissances, des méthodes et des concepts sur le monde non humain, de catalyser en partie le développement de la maturité affective des élèves et de renforcer leur sens de la responsabilité. Conscients de cet enjeu les responsables des programmes d'enseignement pourraient ainsi inscrire cet objectif comme une finalité spécifique à l'enseignement de la biologie.

\section{BIBLIOGRAPHIE}

BEC J.L. \& FAVRE D., « Le système nerveux dans le programme de biologie. Quel(s) concept(s) veut-on enseigner? ", in Actes des $7^{\text {es }}$ Rencontres Européennes de Didactique de la Biologie, Montpellier, 13, 14 et 15 septembre, 1995.

Develay M., De l'apprentissage à l'enseignement, Pour une épistémologie scolaire, E.S.F., 1992.

FAVRE D., «Quelles capacités l'enseignement de biologie permet-il de développer chez les apprenants? », in Actes des $4^{e s}$ Rencontres Européennes de Didactique de la Biologie, (à paraître), Cordoue, 5-7 mai, 1992.

Favre D. \& Rancoule Y., «Peut-on décontextualiser la démarche scientifique ? », in Aster, $\mathrm{N}^{\circ}$ 16,1993, p. 29-46.

LAURET M., « De l'intérêt pour les étudiants en biologie d'être en contact avec leur objet d'étude et son contexte ", in Communication aux $7^{\text {es }}$ Rencontres Européennes de Didactique de la Biologie, Montpellier, 13, 14 et 15 septembre, 1995.

RUMELHARD G., « Le processus de dogmatisation », in Actes des J.I.E.S. I, 1979, p. 11-28.

SEARLES H.F., L'environnement non humain, Paris, Gallimard, (réédition et traduction française 1986), 1960.

STEWART J., "La spécificité épistémologique de la biologie », in Actes des $7^{\text {es }}$ Rencontres Européennes de Didactique de la Biologie, Montpellier, 13, 14 et 15 septembre, 1995.

\section{RÉSUMÉS}

À été appelé : «apparentement » la relation structurante pour sa personnalité qu'un individu tisse avec l'environnement non humain : vivant et / ou non vivant. L'accès à cette "maturité affective » par la médiation de l'enseignement de la biologie paraît, en partie, possible si ce 
dernier parvenait à la fois à nourrir notre lien de parenté avec le monde non humain mais aussi à nous en distancier suffisamment pour devenir apte à se percevoir comme radicalement différent de ce monde.

The word "relatedness" is the term that bas been coined to denote the relationship, constructive to one's personality structure, which an individual fashions "vis à vis" his / her non human environment, be it living or non living. Acceding to this "affective maturity" through the medium of biology instruction appears to be in part possible, if this instruction was able to help us to at one and the came time, develop our close link to the non human world, but also to help us in differentiating ourselves sufficiently from it, so that we may be able to perceive ourselves as fundamentally distinct from the world around us.

\section{INDEX}

Mots-clés : apparentement, enseignement de la biologie, environnement non humain

Keywords : biology teaching, non human environment, relatedness

\section{AUTEUR}

DANIEL FAVRE

Laboratoire de modélisation de la relation pédagogique, équipe ERES, Université de Montpellier II 